\title{
Estudo dos Fatores Sociodemográficos Associados à Aquisição de Infeções Sexualmente Transmissíveis em Estudantes Estrangeiros em Intercâmbio Universitário em Portugal
}

\author{
Study of the Sociodemographic Factors and Risky \\ Behaviours Associated with the Acquisition of Sexual \\ Transmitted Infections by Foreign Exchange Students in \\ Portugal
}

\author{
Andreia GRAVATA ${ }^{1}{ }^{1}$, Rita CASTRO ${ }^{1}$, João BORGES-COSTA ${ }^{1}$ \\ Acta Med Port 2016 Jun;29(6):360-366 - http://dx.doi.org/10.20344/amp.6692
}

\section{RESUMO}

Introdução: As infeções sexualmente transmissíveis são um problema de saúde pública, sendo mais frequentes em jovens. Este estudo teve como principal objetivo avaliar os fatores sociodemográficos e comportamentos associados à aquisição de infeções sexualmente transmissíveis e o conhecimento sobre infeção por Chlamydia trachomatis em estudantes estrangeiros em intercâmbio universitário em Portugal.

Material e Métodos: Os fatores sociodemográficos e comportamentos de risco foram estudados por aplicação de um questionário a estudantes em intercâmbio universitário em Portugal, inscritos nos anos letivos de 2012/2013, 2013/2014 e 2014/2015.

Resultados: Avaliaram-se 338 questionários: 58,3\% participantes do sexo feminino e 40,8\% do sexo masculino (17 aos 30 anos). A idade média apontada para o início da vida sexual foi de 17,5 anos e a média de parceiros sexuais de 6,9 . Relativamente às questões inquiridas foi referido: 9,5\% negaram atividade sexual oral; $29 \%$ com atividade sexual anal; $11,8 \%$ com atividade sexual com parceiros do mesmo género; $82,1 \%$ refere consumo de álcool/estupefacientes; $42,3 \%$ desconhecimento sobre a infeção por Chlamydia trachomatis e $21 \%$ sobre o risco de transmissão de infeção por via oral.

Discussão: Apesar das infeções sexualmente transmissíveis poderem afetar indivíduos de todas as idades, raças e orientações sexuais, vários fatores demográficos, sociais e comportamentais têm revelado influência nas taxas de prevalência deste tipo de infeções.

Conclusões: Nesta população os fatores de risco associados a uma maior prevalência de infeções sexualmente transmissíveis continuam a existir, nomeadamente início precoce da atividade sexual, parceiros sexuais múltiplos e ausência de medidas de proteção durante as relações sexuais.

Palavras-chave: Assunção de Riscos; Chlamydia trachomatis; Comportamento Sexual; Doenças Sexualmente Transmissíveis; Estudantes; Factores Socioeconómicos; Inquéritos e Questionários; Intercâmbio Educacional Internacional; Portugal.

\section{ABSTRACT}

Background: Sexual transmitted infections are a main cause of morbidity, being a public health problem due to its reproductive complications, mostly observed in teenagers and young adults. The purpose of this study was to evaluate sociodemographic factors and risky behaviours associated with sexual transmitted infections acquisition and to assess personal awareness of risky behaviour and the knowledge about Chlamydia trachomatis infection between foreign exchange students in Portugal.

Material and Methods: The main instrument for data collection was a questionnaire, applied to foreign students in university exchange in Portugal, during the years 2012/2013, 2013/2014 e 2014/2015

Results: Three hundred and thirty eight (338) questionnaires were evaluated, being $58.3 \%$ female students, aged between 17 and 30 years old. Mean age for the beginning of the sexual activity was 17.5 years old and the mean number of lifetime sexual partners was 6.9. Concerning the answers given: $11.8 \%$ mentioned a sexual relationship with the same gender, $9.5 \%$ mentioned that they have never done oral sex and $29 \%$ assumed they had practiced anal sex; $82.1 \%$ mentioned alcohol/drugs consumption; $21 \%$ did not know that Sexual transmitted infections can be transmitted through oral sex and $42.3 \%$ did not recognize Chlamydia trachomatis as an Sexual transmitted infections agent.

Discussion: Although sexual transmitted infections can affect individuals of all ages, races and sexual orientation, various demographic, social and behavioral factors have revealed influence in their prevalence rates.

Conclusion: Despite knowing about sexual transmitted infections, these students maintain sexual risky behaviours, mainly early age for starting sexual activity, multiple sexual partners and the absence of protection during sexual activities.

Keywords: Chlamydia trachomatis; International Educational Exchange; Portugal; Risk-Taking; Sexually Transmitted Diseases; Sexual Behavior; Socioeconomic Factors; Students; Surveys and Questionnaires.

\footnotetext{
1. Unidade de Microbiologia Médica. Instituto de Higiene e Medicina Tropical. Universidade NOVA de Lisboa. Portugal.

$\bowtie$ Autor correspondente: Andreia Gravata. andreia.gravata@gmail.com

Recebido: 11 de setembro de 2015 - Aceite: 24 de maio de 2016 | Copyright @ Ordem dos Médicos 2016
} 


\section{INTRODUÇÃO}

As infeções sexualmente transmissíveis (IST) encontram-se entre as causas mais comuns de doença em todo o mundo.$^{1,2}$ Este tipo de infeções têm originado consequências a nível de saúde e da economia, bem como a nível social, em diversos países, ${ }^{2}$ continuando a constituir um problema de saúde pública, pelo risco de transmissão e de desenvolvimento de sequelas, especialmente se não forem diagnosticadas e tratadas precocemente. ${ }^{3}$

Em 1999, a Organização Mundial de Saúde (OMS) estimava que existissem 92 milhões de novos casos de IST. No entanto, com o aumento previsto das notificações, as últimas estimativas indicam a existência anual de 498.9 miIhões de novos casos de IST em indivíduos com idades compreendidas entre os 15 e os 49 anos. ${ }^{4}$

Os adolescentes e os jovens adultos (grupos etários definidos pela OMS com idades compreendidas entre os $10 \mathrm{e}$ os 19 anos e os 15 e os 24 anos, respetivamente) são os grupos etários de maior risco para a aquisição de uma IST, ${ }^{5}$ facto suportado por uma combinação de fatores comportamentais, biológicos e culturais. ${ }^{6}$

A infeção por Chlamydia trachomatis é a infeção bacteriana sexualmente transmitida mais comum em todo o mundo, ${ }^{7,8}$ existindo uma forte associação entre as taxas de infeção mais elevadas e as faixas etárias mais jovens. ${ }^{9}$ Esta, tem vindo a aumentar em muitos países, e o seu caracter assintomático (cerca de $70-85 \%$ das mulheres e $50 \%$ dos homens) torna-a uma preocupação global a nível de saúde pública. ${ }^{10}$ Apesar da subnotificação associada a esta infeção, estima-se que na Europa a sua prevalência oscile entre os $5 \%$ e os $15 \%,{ }^{1}$ com as maiores taxas de incidência em jovens com idades inferiores aos 25 anos. ${ }^{1,7}$

Este trabalho teve como objetivo o estudo dos fatores sociodemográficos e comportamentais associados à aquisição de IST, permitindo identificar os fatores de risco pessoal para aquisição de IST e o conhecimento sobre a infeção por $C$. trachomatis por parte dos estudantes estrangeiros em intercâmbio universitário em Portugal.

\section{MATERIAL E MÉTODOS}

O presente estudo foi submetido e aceite pela Comissão de Ética do Instituto de Higiene e Medicina Tropical (IHMT) da Universidade Nova de Lisboa. A amostra alvo foi constituída por estudantes em intercâmbio universitário em Portugal, matriculados em Instituições do Ensino Superior nos anos letivos 2012/2013, 2013/2014 e 2014/2015, com idade igual ou inferior a 30 anos de idade e que já tivessem iniciado a vida sexual.

A elaboração deste trabalho incluiu, por um lado, a distribuição de um questionário presencial aos estudantes universitários em intercâmbio em instituições do Ensino Superior da área de Lisboa e, por outro lado, o envio por correio eletrónico do mesmo questionário em formato online a estudantes estrangeiros inscritos em 25 instituições de ensino superior em Portugal. Para o preenchimento do questionário presencial, os estudantes foram contactados nos cursos de Língua Portuguesa para estrangeiros lecionados na Faculdade de Ciências Sociais Humanas, Universidade Nova de Lisboa, nas redes sociais (essencialmente através da utilização do site www.facebook.com) e no Hospital de Santa Maria - Centro Hospitalar Lisboa Norte E.P.E., durante o estágio de Medicina nas consultas de Dermatologia. Nestes casos, foi apresentado aos estudantes uma breve explicação sobre a temática e os objetivos do estudo, e os que aderiram assinaram uma declaração de consentimento informado. Para o envio do questionário online, utilizou-se um software gratuito de questionários e inquéritos (survio. com). Este questionário (acesso através de um link direto), juntamente com um texto explicativo sobre o projeto, elaborados em inglês, foi enviado via correio eletrónico aos alunos estrangeiros através dos gabinetes de relações internacionais das 25 instituições que se disponibilizaram para colaborar (Apêndice em: http://www.actamedicaportuguesa.com/revista/index.php/amp/article/view/6692/360366_Apendice01.pdf).

Ambos os questionários, devido à sensibilidade do tema abordado, garantiram anonimato e confidencialidade, permitindo aos estudantes optar por não facultar qualquer tipo de resposta.

$\mathrm{Na}$ análise estatística dos resultados utilizou-se o programa estatístico IBM ${ }^{\circledast}$ SPSS $^{\circledast}$ (Statistical package for the social sciences, versão 21 , SPSS Inc, Chicago, IL, USA). Além da análise exploratória e estatística descritiva dos dados, foram efetuados alguns testes de hipóteses, com um nível de significância de 5\%.

\section{RESULTADOS}

Trezentos e trinta e oito questionários, 167 preenchidos presencialmente e 171 preenchidos online, foram obtidos.

Os participantes deste estudo tinham idades compreendidas entre os 17 e os 30 anos (média de 23,17 e desvio padrão de 2,40 ), sendo $58,3 \%$ do sexo feminino e $40,80 \%$ do sexo masculino; três participantes não responderam a esta questão $(0,9 \%)$.

As nacionalidades dos participantes foram muito diversas, existindo estudantes de 46 nacionalidades diferentes, destacando-se como mais frequentes as nacionalidades italiana $(23,40 \%, 79 / 338)$, espanhola $(14,50 \%, 49 / 338)$, alemã $(9,8 \%, 33 / 338)$ e brasileira $(7,4 \%, 25 / 338)$.

Relativamente à idade do início da atividade sexual, responderam 319/338 (94,38\%), tendo-se verificado que a idade média foi de 17,48 anos (desvio padrão 2,36). Do total analisado, 5,96\% (19/319) iniciaram a atividade sexual com idade igual ou inferior a 14 anos e 44,20\% (141/319) com idade igual ou superior a 18 anos. Na análise estatística, não se verificaram diferenças estatisticamente significativas para as idades de início de atividade sexual e o género.

A média de parceiros sexuais desde o início da atividade sexual dos participantes no estudo foi de 6,87 (desvio padrão de 7,52) e 57,70\% (195/338) dos estudantes referiu entre um a cinco parceiros. O número médio de parceiros sexuais dos participantes do sexo feminino foi inferior ao 
Tabela 1 - Média de parceiros sexuais totais por idade início das atividades sexuais.

\begin{tabular}{lc}
\hline Idade do início da atividade sexual (anos) & $\mathbf{N}^{\circ}$ médio de parceiros sexuais \\
\hline$\leq 14$ & 13,26 (desvio padrão 15,92) \\
{$[15-17]$} & $7,98$ (desvio padrão 7,04$)$ \\
$\geq 18$ & 5,14 (desvio padrão 5,76 ) \\
\hline
\end{tabular}

$\rho<0,05$ no testes estatístico não paramétrico de Kruskal-Wallis (valor de significância de 0,05 )

número médio obtido para os do sexo masculino $(6,01 \mathrm{e}$ 8,11 , respetivamente), correspondendo a uma diferença estatisticamente significativa, suportada pela aplicação do teste de Mann-Whitney. Relativamente ao número de parceiros sexuais nos seis meses anteriores ao estudo, a média foi de 1,89 (desvio padrão de 2,07) sendo de realçar que $52,07 \%$ (60,79\% [107/176] do sexo feminino e $39,20 \%$ [69/176] do sexo masculino) referiram ter tido apenas um parceiro.

Aplicado o teste de Kruskal-Wallis, verificou-se a existência de uma diferença significativa entre a idade de início das relações sexuais e o número médio total de parceiros sexuais (Tabela 1), tendo-se registado uma média de parceiros sexuais superiores no grupo de jovens que iniciou a atividade sexual mais precocemente (com 14 anos ou menos) comparativamente ao grupo que iniciou entre os $15 \mathrm{e}$ os 17 anos e o grupo que iniciou aos 18 anos ou mais tarde (tendo este último grupo o valor médio mais baixo).

Quando questionados em relação à utilização do preservativo em relações sexuais vaginais/anais, $67,77 \%$ (225/332) dos estudantes referiu a sua utilização, não havendo diferenças significativas entre género. Dos que referiram não utilizar preservativo, 50,47\% (54/107) não recorre a quaisquer outros métodos anticoncecionais.

Em relação à orientação e comportamentos sexuais verificou-se que $11,98 \%$ (40/334) estudantes indicaram atividade sexual com parceiros do mesmo género. Destes, $60 \%$ (24/40) relataram a prática exclusiva de homossexualidade e $40 \%(16 / 40)$ de bissexualidade. Apenas 9,64\% (32/332) dos estudantes referiram não praticar relação sexual oral, tendo-se observado uma associação estatisticamente significativa entre este tipo de relação sexual e o género, já que as estudantes do sexo feminino negaram mais frequentemente do que os estudantes do sexo masculino este tipo de relação sexual (27/32 e 5/32, respetivamente). Na questão sobre relação sexual do tipo anal, $29,70 \%$ dos estudantes (98/330) responderam afirmativamente, havendo uma tendência para esta questão ser mais frequentemente negada entre o género feminino comparativamente ao género masculino $(64,20 \%$ e $35,80 \%$, respetivamente). Dos estudantes que admitiram efetuar relações sexuais orais e anais, $95 \%$ (285/300) e 37,70\% (36/98) respetivamente, afirmaram não utilizar o preservativo durante as mesmas.

O consumo de álcool e de estupefacientes foi admitido por $82,10 \%(266 / 324)$ e $32,13 \%(107 / 333)$ dos jovens respetivamente, tendo a maioria $(70,48 \%$ - $234 / 332)$, assumido já ter tido relações sexuais sob o efeito do álcool e 19,28\% (64/332) referiu ainda a utilização de estupefacientes (em especial marijuana - Cannabis sativa) antes ou durante o ato sexual, não havendo diferenças significativas entre os géneros em nenhum dos casos.

De todos os estudantes que facultaram resposta, $7,83 \%$ (26/332) mencionaram aquisição anterior de uma infeção sexualmente transmissível: infeção genital por $C$. trachomatis $(1,8 \%)$, condilomas genitais $(1,2 \%)$, herpes genital $(0,6 \%)$, gonorreia $(0,3 \%)$, VIH (vírus da imunodeficiência humana, $0,3 \%$ ), havendo dois casos de coinfecção por dois agentes (papilomatose/candidose vaginal e herpes genital/ candidose vaginal). Apenas $35,84 \%$ (119/332) referiram ter efetuado rastreio de infeção por VIH nos últimos cinco anos.

Relativamente às questões que pretenderam avaliar o conhecimento sobre IST, $21,02 \%$ (70/333) dos estudantes admitiu não conhecer o risco de transmissão de infeção por via oral e 42,34\% (141/333) assumiu não ter o conhecimento prévio sobre a infeção por Chlamydia trachomatis. Nesta questão, verificou-se que no grupo de estudantes que referiu já ter tido uma IST anteriormente o conhecimento prévio sobre esta infeção tendeu a ser superior.

Para o grupo de estudantes que respondeu ao questionário online, tentou-se também avaliar a taxa de adesão a um rastreio de infeção por $C$. trachomatis gratuito e verificou-se que apenas $42,70 \%$ (73/171) mostraram interesse em efetuar o rastreio, enquanto 13,50\% (13/171) não facultou resposta. Neste contexto, não foi encontrada qualquer associação com a área de estudo, género ou idade da primeira relação sexual, no entanto, verificou-se uma maior predisposição para efetuar o rastreio em jovens com história anterior de IST, comparativamente aos que não tiveram diagnóstico prévio de uma IST (confirmado pelo teste de Fisher).

\section{DISCUSSÃO}

Apesar das IST poderem afetar indivíduos de todas as idades, raças e orientações sexuais, vários fatores demográficos, sociais e comportamentais têm revelado influência nas taxas de prevalência deste tipo de infeções. ${ }^{10} \mathrm{~A}$ idade inferior a 25 anos, estar solteiro(a), ter mais do que três parceiros sexuais no prazo de três meses ou um novo parceiro sexual num prazo de três meses, ${ }^{10,11}$ ter um parceiro sexual com IST ${ }^{12}$ ou que recorra ao contacto sexual com múltiplos parceiros, ${ }^{1}$ o elevado consumo de álcool e de drogas e a fraca adesão à utilização do preservativo, são alguns exemplos. ${ }^{10-12}$

De uma forma geral, e tal como se verificou neste estudo, em trabalhos de investigação sobre educação e saúde sexuais, tem-se verificado uma maior adesão à participação por parte do género feminino, ${ }^{7} \mathrm{em}$ parte, devido ao facto do 
sexo masculino continuar a acreditar ser esta uma temática pertencente a um público exclusivamente feminino. ${ }^{13}$ Por outro lado, são também as mulheres que recorrem a consultas de acompanhamento ginecológico e de planeamento familiar, o que poderá deixá-las mais sensibilizadas para as questões relacionadas com a saúde reprodutiva e sexual. ${ }^{13}$

Nos Estados Unidos da América (EUA) e em Inglaterra, vários estudos têm indicado a existência de uma associação entre o início precoce da atividade sexual (10-14 anos) e a tendência a assumir comportamentos de risco na aquisição de IST, nomeadamente, e tal como se verificou neste estudo, no que diz respeito ao elevado número de parceiros sexuais ao longo da vida. ${ }^{14}$ Segundo o Centers for Disease Control and Prevention (CDC), os jovens que iniciam as relações sexuais precocemente estão incluídos num grupo de alto risco de aquisição de IST, principalmente devido ao elevado número de parceiros sexuais, ao desenvolvimento de relações de curta duração, à fraca adesão à utilização de barreiras contracetivas, à predisposição biológica para aquisição de infeções (especialmente em mulheres jovens), e ainda à reduzida procura dos serviços de saúde. ${ }^{15}$ Esta questão tem-se tornado cada vez mais importante, uma vez que vários estudos têm encontrado uma tendência gradual para a diminuição da média de idades para o início da atividade sexual. ${ }^{14}$ Muitos jovens sexualmente ativos afirmam terem sentido alguma pressão social para iniciarem a sua vida sexual a partir de determinada idade e mais de metade concorda que uma vez iniciada a vida sexual, torna-se difícil recusar a prática sexual em futuras relações, especialmente nos indivíduos do sexo masculino, género onde o início da atividade sexual é mais precoce (comparativamente ao sexo feminino com a mesma idade). ${ }^{16}$ No presente estudo a associação entre o género e o início precoce da atividade sexual não foi estatisticamente confirmada.

Ainda que a comparação com outros estudos semeIhantes tenha sido difícil, uma vez que a maioria tem em conta o número de parceiros sexuais em períodos de tempo de 12 meses em vez de seis meses, a média de parceiros sexuais no nosso grupo de estudo foi superior à encontrada na literatura. ${ }^{17}$

Apesar de mais de metade $(52,1 \%)$ dos estudantes ter referido o contacto sexual com apenas um parceiro sexual nos últimos seis meses, o rastreio de infeção por $C$. trachomatis nestes casos de aparente baixo risco, deverá ser proposto. Algumas IST, nomeadamente a supracitada, são relativamente frequentes tanto em indivíduos com poucos parceiros sexuais como em indivíduos com múltiplos parceiros sexuais (ainda que estes últimos estejam sob maior risco de infeção). ${ }^{18}$

Relativamente às relações homossexuais e/ou bissexuais, verificou-se que a percentagem de jovens a referir ter tido relações sexuais com indivíduos do mesmo sexo foi semelhante às encontradas em outros estudos. ${ }^{19}$ Neste contexto, e segundo o CDC, os homens que têm sexo com outros homens $(\mathrm{HSH})$ têm um risco acrescido de aquisição de IST, principalmente devido à troca frequente de parceiro sexual, elevadas taxas de contactos sexuais casuais e à utilização de drogas antes e no decorrer da relação sexual. ${ }^{5}$

A utilização do preservativo masculino é um método preventivo extremamente eficaz contra aquisição de IST, uma vez que, quando usado corretamente constitui uma barreira física que impossibilita a passagem dos agentes causadores de infeção. ${ }^{20} \mathrm{~A}$ baixa adesão à utilização do preservativo e a crença por parte dos jovens de que a utilização de métodos contracetivos, que não o preservativo, é suficientemente segura (ainda que estes não assegurem proteção contra as IST) mostram que os jovens poderão não estar totalmente conscientes de que ter atividade sexual sem proteção poderá colocá-los em risco de adquirir uma IST. ${ }^{21}$ De uma forma geral, os valores encontrados em diversos estudos para a utilização do preservativo variam fortemente com os critérios de avaliação utilizados. O presente estudo apresentou taxas de utilização do preservativo idênticas às de alguns estudos efetuados nos EUA. ${ }^{7,21}$ Salienta-se que a maioria destes estudos, não tem em conta a distinção entre relação sexual oral, anal e vaginal, sendo de assinalar a elevada quantidade de jovens que tem relações sexuais orais sem utilização de preservativo $(95 \%)$. Um trabalho desenvolvido pela Henry J. Kaiser Family Foundation demonstrou que muitos jovens, questionados sobre os motivos que os levam a rejeitar a utilização do preservativo, consideram que a abordagem acerca da utilização do preservativo com o parceiro sexual pode levantar suspeitas sobre a sua própria história sexual ou sobre o passado sexual do parceiro, ${ }^{22}$ e um em cada dez jovens americanos admite sentir-se insultado pela sugestão de utilizar preservativo durante a relação sexual. ${ }^{22}$ Alguns jovens afirmam ainda sentirem-se desconfortáveis e envergonhados durante a compra do preservativo, sublinhando a dificuldade pessoal em abordar assuntos relacionados com a contraceção, mesmo com profissionais de saúde..$^{22}$ Aqueles, preferem adotar outros métodos, particularmente o anticoncetivo oral e o coito interrompido ou a ejaculação externa como métodos de contraceção, ainda que estes não garantam proteção adequada quer contra uma gravidez não desejada, quer contra IST. ${ }^{22}$

Atualmente tem havido referências à existência de uma associação entre o consumo de álcool/drogas e o aumento da frequência das relações sexuais e do número de parceiros sexuais, através da diminuição das inibições e consequente aumento dos comportamentos de risco. ${ }^{1,10}$ Neste trabalho e comparativamente a outros do mesmo género, verificou-se que o consumo de estupefacientes referente aos últimos seis meses foi idêntico e que o consumo de álcool foi superior. ${ }^{7} \mathrm{O}$ consumo de drogas, fator associado à reduzida utilização do preservativo e consequente aumento do risco de aquisição de IST, tem vindo a aumentar desde a década de noventa do século passado, sendo o mais frequente entre os jovens, após o consumo de álcool e de tabaco. ${ }^{12}$

Segundo alguns autores a educação sexual é importante, especialmente em jovens com história anterior de 
IST, uma vez que uma experiência anterior poderá deixar os jovens mais atentos a comportamentos de risco e ao aparecimento de sintomas sugestivos de infeção. ${ }^{21}$ Apesar da incidência de IST anterior nos participantes deste estudo ter sido reduzida (comparativamente a outros estudos),${ }^{16}$ verificou-se que os estudantes que tiveram contacto prévio com IST apresentaram uma maior tendência para reconhecer a infeção por C. trachomatis. Ainda assim, a literatura continua a revelar que grande parte dos jovens tendem a subestimar o risco de adquirir infeções, mesmo quando no passado Ihes foi diagnosticada uma IST. ${ }^{21}$ E tem-se verificado também que apesar do conhecimento por parte dos jovens sobre as IST, os fatores de risco associados a maiores prevalências de infeção, mantêm-se constantes, nomeadamente o início precoce da atividade sexual, os parceiros sexuais múltiplos e a ausência de medidas de proteção durante as relações sexuais.

Um quinto dos estudantes que respondeu ao inquérito assumiu não estar consciente do risco de aquisição de uma IST através de uma relação sexual oral, o que está de acordo com o encontrado em outras publicações, as quais inclusive sublinham, que os jovens acreditam que este tipo de relação é a mais segura e eficaz como método preventivo de gravidez e de IST. ${ }^{22}$ Outros autores revelam a existência de lacunas e ideias erradas no conhecimento geral dos jovens sobre as IST: nos EUA um quinto dos jovens acredita conseguir detetar a presença de uma IST no parceiro, mesmo sem a aplicação de um teste de diagnóstico, e um sexto dos jovens admite estar sob risco de aquisição de IST apenas se estiver presente sintomatologia óbvia. ${ }^{22}$

Muitos jovens continuam a desconhecer a infeção por C. trachomatis, ${ }^{14}$ e apesar de $42,34 \%$ dos estudantes desta análise ter referido não ter conhecimento prévio sobre a infeção por aquele agente, vários autores mencionam que este desconhecimento afeta mais de metade dos jovens. Neste contexto, o facto da população alvo ter um nível de escolaridade superior ao de muitos estudos semelhantes aplicados em jovens, poderá ter influenciado as taxas de conhecimento de IST. ${ }^{7}$

Não se encontraram diferenças significativas relativamente ao conhecimento sobre a infeção por $C$. trachomatis entre rapazes e raparigas participantes no presente estudo, no entanto tem-se verificado na literatura haver um maior conhecimento por parte do sexo feminino, o que poderá estar relacionado com a maior adesão por parte das raparigas a estudos relacionados com as IST. ${ }^{13}$ Para esta questão, também não foram encontradas diferenças significativas entre o grupo que respondeu presencialmente e o grupo que respondeu online, apesar da possibilidade de pesquisa online sobre as IST no segundo grupo. Ainda assim, e independentemente da fonte de informação sobre a saúde sexual, mais de três quartos dos jovens têm expressado, em outros estudos, a necessidade de obter mais informação sobre tópicos sexuais, dada a importância de inúmeras decisões no âmbito do relacionamento sexual. ${ }^{22}$

Na Europa e no resto do mundo, a taxa de estudantes relutantes em participar em rastreios gratuitos é muito elevada, apesar do tipo de colheita ser não invasivo., ${ }^{7,13,23}$ O processo de rastreio, referente à utilização de testes de diagnóstico laboratorial em indivíduos sem sintomatologia, para o diagnóstico e respetivo tratamento das infeções assintomáticas, possibilita não só a redução do tempo de infeção, como conduz à menor probabilidade de aparecimento de complicações e à diminuição do tempo de risco de contágio. ${ }^{18}$ Nesta perspetiva, o CDC e o USPST (United States Preventive Services Task Force - EUA), recomendam a aplicação de rastreios anuais a todas as mulheres sexualmente ativas com idades inferiores aos 25 anos, a mulheres com fatores de risco aumentados (novo parceiro sexual ou parceiros sexuais múltiplos), e ainda em $\mathrm{HSH}$ (testes para infeção uretral, retal e faríngea, sempre que se justifique). ${ }^{5}$

Vários autores têm focado o seu objetivo de estudo na deteção das principais barreiras à realização de rastreios voluntários e, de um modo geral, têm justificado essa baixa adesão com vários fatores, tais como o desconhecimento, por parte dos jovens, de estar em risco, ${ }^{14,23}$ o reduzido nível de conhecimento público de infeção por C. trachomatis, ${ }^{13}$ a falta de conhecimento do que envolve e de como aceder aos testes de diagnóstico (receio da aplicação de técnicas invasivas ${ }^{8,14}$ ou de técnicas de deteção de drogas ${ }^{14}$ ou o acreditar de que estes exames estão incluídos nos exames médicos de rotina), ${ }^{22}$ a natureza assintomática das infeções, as falsas certezas associadas à atividade sexual (nomeadamente a associação desta IST a atividades sexuais "menos próprias" ou a um público exclusivamente feminino $)^{13}$ e ainda a existência de constrangimentos e estigmas relacionados com a temática sexual. ${ }^{14,23}$

Tendo como base as baixas taxas de adesão a testes de rastreio, nomeadamente infeção por $C$. trachomatis, por parte da população jovem, é de extrema importância monitorizar o grau de conhecimento sobre esta bactéria, de forma a perceber qual o melhor modo de chegar à população jovem e promover a importância da sua saúde sexual. É de reconhecimento geral que as abordagens convencionais para os rastreios, aplicadas em serviços de saúde, não têm sido eficazes, e poderá ser importante adotar novos tipos de programas de rastreio, feitos numa base mais oportunista, com a aplicação de rastreios não só em serviços de saúde, mas também em farmácias, através de auto colheita (via internet ou por correio) ou até em escolas. Em Inglaterra, esta nova abordagem tem demonstrado a capacidade de atingir elevada cobertura populacional, com cerca de 59\% dos diagnósticos em jovens dos 15 aos 24 anos aplicados fora de clínicas especializadas em medicina geniturinária.

A existência de uma relação estatística entre os baixos níveis de educação e o aumento dos comportamentos de risco de aquisição de IST tem sido observada em estudos anteriores. ${ }^{7} \mathrm{Na}$ população do presente estudo, mesmo em indivíduos com níveis de escolaridade elevados, verificou-se que os comportamentos de risco continuam a existir em grande escala (especialmente ao nível de consumo de álcool/drogas, da fraca utilização do preservativo e da fraca adesão aos testes de rastreio), e tal poderá ser resultado 
de uma vida académica que proporciona melhores oportunidades para enveredar por práticas de risco. ${ }^{19}$ Para além disso, e segundo a OMS, as populações migratórias estão sob maior risco de aquisição de IST, ${ }^{20}$ sendo que estes estudantes, pelo menos durante um período de tempo, estarão fora do seu país e das suas rotinas normais.

As limitações deste estudo passam essencialmente pela natureza estigmática que atualmente ainda acompanha a abordagem da temática sexual, conduzindo à recusa de colaboração na divulgação dos questionários pelos alunos por parte de várias instituições do Ensino Superior. Para além disso, a existência de um questionário online poderá ter propiciado aos participantes a prestação de falsas declarações.

\section{CONCLUSÕES}

A fase universitária favorece o aparecimento e consolidação de determinados comportamentos de risco (especialmente a nível de consumo de álcool/drogas, da fraca utilização do preservativo e a fraca adesão a testes de rastreio), mesmo em indivíduos com níveis de escolaridade elevados. No entanto, estes comportamentos não são percecionados pelos estudantes como de risco, colocando-os em elevado risco de adquirir infeção por C. trachomatis, assim como outras IST.

Tendo como base as baixas taxas de adesão aos testes de rastreio, nomeadamente de $C$. trachomatis, por parte da população jovem, é de extrema importância monitorizar o grau de conhecimento sobre esta bactéria, de forma a perceber qual o melhor modo de chegar à população jovem e de promover a noção da importância da sua saúde sexual.

A educação sexual é essencial para a promoção da alteração comportamental, da prevenção de infeções e para auxiliar na utilização dos serviços de saúde de forma mais frequente. Assim, é importante a garantia de que os

\section{REFERÊNCIAS}

1. Gross G, Tyring SK. Sexually transmitted infections and sexually transmitted diseases. New York: Springer; 2011.

2. World Health Organization. Global Strategy for the Prevention and Control of Sexually Transmitted Infections: 2006-2015: Breaking the chain of transmission. Geneva: WHO; 2007.

3. Centers for Disease Control and Prevention. Incidence, Prevalence, and Cost of Sexually Transmitted Infections in the United States. Fact Sheet; 2013. [consultado 2015 jul 13]. Disponível em: http://www.cdc.gov/std/ stats/

4. World Health Organization: Global prevalence and incidence of selected curable sexually transmissible disease: overview and estimates. Geneva: WHO; 2001.

5. Centers for Disease Control and Prevention. Chlamydia - Fact Sheet, 2011. [consultado 2015 jul 15]. Disponível em: http://www.cdc.gov/

6. Centers for Disease Control and Prevention. Sexually Transmitted Disease Surveillance 2011. Atlanta: Department of Health and Human Services; 2012.

7. Spauwen LL, Hoebe CJ, Brouwers EE, Dukers-Muijrers NH. Improving STD testing behavior among high-risk young adults by offering STD testing at a vocational school. BMC Public Health. 2011;11:750.

8. Walker J, Tabrizi SN, Fairley CK, Chen MY, Bradshaw CS. Chlamydia trachomatis incidence and re-infection among young women behavioural and microbiological characteristics. PLoS ONE. 2012;7:1-9.

9. Morré SA, Brule VD, Adriaan JC, Lawrence R, Boeke AJP, Voorhorst $\mathrm{FJ}$, et al. The natural course of asymptomatic Chlamydia trachomatis infections: $45 \%$ clearance and no development of clinical PID after oneyear follow-up. Int J STD AIDS. 2002;13:12-8. jovens recebem informação sobre as IST: modo de transmissão, métodos de prevenção e sobre a importância do tratamento completo para evitar reinfeções.

É ainda essencial a implementação da notificação dos parceiros sexuais dos indivíduos infetados, encorajando-os à execução dos testes de rastreio, bem como do seu tratamento adequado, já que as taxas de reinfeção são elevadas e cruciais na disseminação das IST.

\section{Observações}

Resumo apresentado nas 6as. Jornadas do IHMT, 11 de Dezembro de 2015. Andreia GRAVATA et al. Estudo de fatores sociodemográficos associados à aquisição de IST em estudantes estrangeiros em intercâmbio universitário em Portugal.

\section{PROTECÇÃO DE PESSOAS E ANIMAIS}

Os autores declaram que os procedimentos seguidos estavam de acordo com os regulamentos estabelecidos pelos responsáveis da Comissão de Investigação Clínica e Ética e de acordo com a Declaração de Helsínquia da Associação Médica Mundial.

\section{CONFIDENCIALIDADE DOS DADOS}

Os autores declaram ter seguido os protocolos do seu centro de trabalho acerca da publicação de dados.

\section{CONFLITO DE INTERESSES}

Os autores declaram não ter nenhum conflito de interesses relativamente ao presente artigo.

\section{FONTES DE FINANCIAMENTO}

Não existiram fontes externas de financiamento para a realização deste artigo.

10. Fanfair R, Workowsky KA. Clinical update in sexually trasnmitted diseases - 2014. Cleveland Clin J Med. 2014;81:91-101.

11. World Health Organization. Guidelines for the management of sexually transmitted infections. Geneva: WHO; 2003.

12. Borges-Costa J: Infeções sexualmente transmissíveis em adolescentes: prevalência e associação com fatores sociodemográficos. [Tese apresentada para a obtenção do grau de Doutor em Ciências Biomédicas. Lisboa: Universidade Nova de Lisboa; 2009.

13. Lorimer K. Hart G. Knowledge of Chlamydia trachomatis among men and women approached to participate in community-based screening, Scotland, UK. BMC Public Health. 2010;10:794.

14. Toby Lea. Chlamydia and young people. Soc Res Briefs. 2010;17:1-4.

15. Workowski KA, Berman S; Centers for Disease Control and Prevention (CDC). Sexually transmitted diseases treatment guidelines, 2010. MMWR Recomm Rep. 2010;59(RR-12):1-110.

16. Lee SJ, Cho YH, Ha US, Kim SW, Yoon MS, Bae K. Sexual behavior survey and screening for chlamydia and gonorrhea in university students in South Korea. Int J Urol. 2005;12:187-93.

17. Lewis D, Newton DC, Guy RJ, Ali H, Chen MY, Fairley CK, et al. The prevalence of Chlamydia trachomatis infection in Australia: a systematic review and meta-analysis. BMC Infect Dis. 2012;12:113.

18. Public Health England. OOpportunistic Chlamydia screening of young adults in England: an evidence summary. 2014. [consultado 2015 jun 23]. Disponível em: http://www.gov.uk/phe

19. Ehis OL, Cyril O, Tolulope E, Deborah OF. Prevalence and risk of Chlamydia trachomatis in symptomatic patients attending clinics in South West Nigeria. J Clin Microbiol. 2014; 5: 1-11. 
20. World Health Organization. Sexually transmitted and other reproductive tract infections: a guide to essential practice. Geneva: WHO; 2005.

21. Ethier KA, Kershaw T, Niccolai L, Lewis JB, Ickovics JR. Adolescent women underestimate their susceptibility to sexually transmitted infections. Sex Transm Infect. 2003;79:408-41.

22. National Survey of Adolescents and Young Adults. Sexual health knowledge, attitudes and experiences. Oakland: Henry J. Kaiser Family Foundation; 2003.

23. Rogstad KE, Bates SM, Partridge S, Kudesia G, Poll R, Osborne MA, et al. The prevalence of Chlamydia trachomatis infection in male undergraduates: a postal survey. Sex Transm Inf. 2001;77:111-3. 